\title{
Keynote: Performance, Availability and Security in Web Design
}

\author{
Virgilio A.F. Almeida \\ Federal University of Minas Gerais - UFMG \\ Brazil \\ virgilio@dcc.ufmg.br
}

\section{Brief Bio}

Virgilio A.F. Almeida is a Professor and former Chair of the Computer Science Department at the Federal University of Minas Gerais (UFMG), Brazil. He received a Ph.D. degree in Computer Science from Vanderbilt University, an MS in Computer Science from the Pontifical Catholic University in Rio de Janeiro (PUC-RIO), and BSEE from UFMG. His research interests include analysis, modeling, and evaluation of the behavior of large scale distributed systems, capacity planning, IT security, Internet and WWW technologies. He was a Visiting Professor at Boston University (1996) and Polytechnic University of Catalonia in Barcelona (2003) and held visiting appointments at Xerox PARC (1997) and Hewlett-Packard Research Laboratory in Palo Alto (2001 and 2004).

He has published over 100 technical papers and was co-author of five books, including "Performance by Design: computer capacity planning by example" "Capacity Planning for Web Services: metrics, models, and methods," "Scaling for E-business: technologies, models, performance, and capacity planning," and "Capacity Planning and Performance Modeling: from mainframes to client-server systems", published by Prentice Hall in 2004, 2002, 2000, and 1994, respectively. He is also a frequent reviewer for a variety of international journals covering Internet and distributed systems issues, and served on the programme committees of various international conferences (including the WWW Conference, ACM-SIGMETRICS, and Performance 2005). He was the program co-chair of the ACM-WOSP'2004 Conference and will be the general co-chair of ACMUSENIX IMC 2006 Conference. Almeida serves on the Editorial Board of First Monday, a multidisciplinary journal on the Internet (www.firstmonday.org).

His research has been funded by the Brazilian Research Council (CNPq), Brazilian Ministry of Science and Technology, and HP Brazil. Almeida was the recipient of various prizes, teaching awards, and best paper awards including an award from Compaq/Brazil for the paper "Characterizing Reference Locality in the WWW" (with Mark Crovella and Azer Bestavros). He served as the chairman of the National Board on Graduate Education in Computer Science in Brazil and as a member of the Computer Science Committee in the Brazilian Research Council. 


\section{Talk Abstract}

Performance, around-the-clock availability, and security are the most common indicators of quality of service on the Web. Management faces a twofold challenge. On the one hand, it has to meet customer expectations in terms of quality of service. On the other hand, companies have to keep IT costs under control to stay competitive. Planning the infrastructure of Web services requires more than just adding extra hardware or software. It requires more than intuition, ad-hoc procedures, or rules of thumb. Many possible alternative solutions can be used to implement a Web service; one has to be able to determine the most cost-effective system solution. This is where Web Engineering and Capacity Planning techniques come into play. This presentation introduces quantitative capacity planning techniques and examples for different Web scenarios, showing precisely how to identify and address performance, availability and security-related problems. 